Erratum

\title{
Erratum to "The Real and Complex Hermitian Solutions to a System of Quaternion Matrix Equations with Applications"
}

\author{
Shao-Wen Yu \\ Department of Mathematics, East China University of Science and Technology, Shanghai 200237, China \\ Correspondence should be addressed to Shao-Wen Yu; yushaowen@ecust.edu.cn \\ Received 11 September 2014; Accepted 23 September 2014; Published 30 September 2014 \\ Copyright (c) 2014 Shao-Wen Yu. This is an open access article distributed under the Creative Commons Attribution License, which \\ permits unrestricted use, distribution, and reproduction in any medium, provided the original work is properly cited.
}

In Lemma 2.4, the equalities in (2.7) and (2.8) should be deleted. Before Lemma 2.4, the following statement should be added.

Following the main idea of the proofs of Theorems 2.1 and 3.1 in [1], we can derive the following lemma over $\mathbb{H}$.

The original Lemma 2.4 should be replaced by the following.

Lemma 2.4. Let $A=A^{*} \in \mathbb{T}^{m \times m}, B \in \mathbb{T}^{m \times n}$, and $C \in \mathbb{T}^{p \times m}$ be given; if $\mathscr{R}(B)$ is contained in $\mathscr{R}\left(C^{*}\right)$, then

$$
\begin{gathered}
\max _{X} r\left[A-B X C-(B X C)^{*}\right]=\min \left\{r\left[A C^{*}\right], r\left[\begin{array}{cc}
A & B \\
B^{*} & 0
\end{array}\right]\right\}, \\
\min _{X} r\left[A-B X C-(B X C)^{*}\right] \\
=2 r\left[A C^{*}\right]+r\left[\begin{array}{cc}
A & B \\
B^{*} & 0
\end{array}\right]-2 r\left[\begin{array}{cc}
A & B \\
C & 0
\end{array}\right] .
\end{gathered}
$$

\section{References}

[1] Y. Liu and Y. Tian, "A simultaneous decomposition of a matrix triplet with applications," Numerical Linear Algebra with Applications, vol. 18, no. 1, pp. 69-85, 2011. 


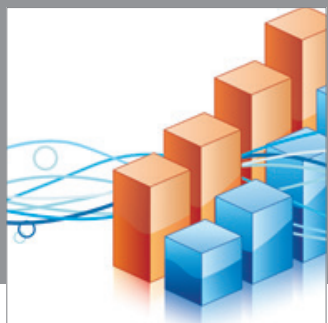

Advances in

Operations Research

mansans

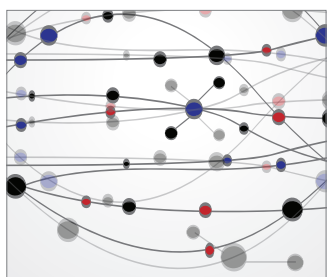

The Scientific World Journal
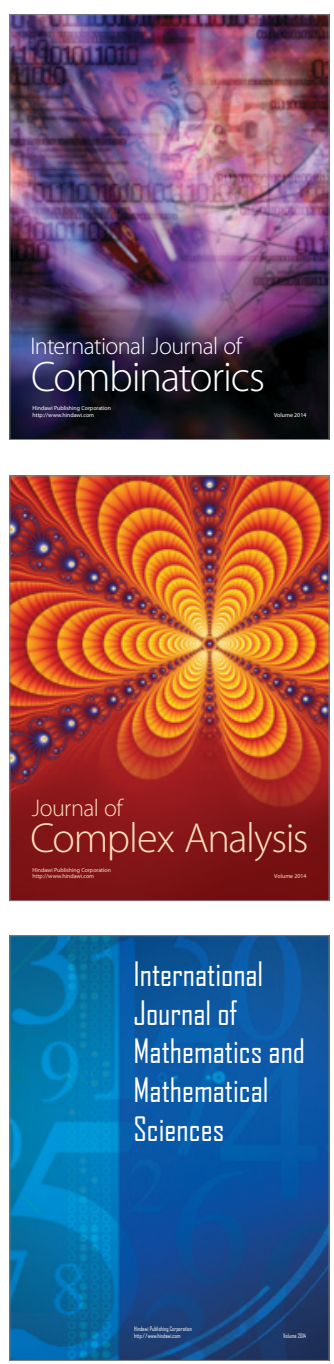
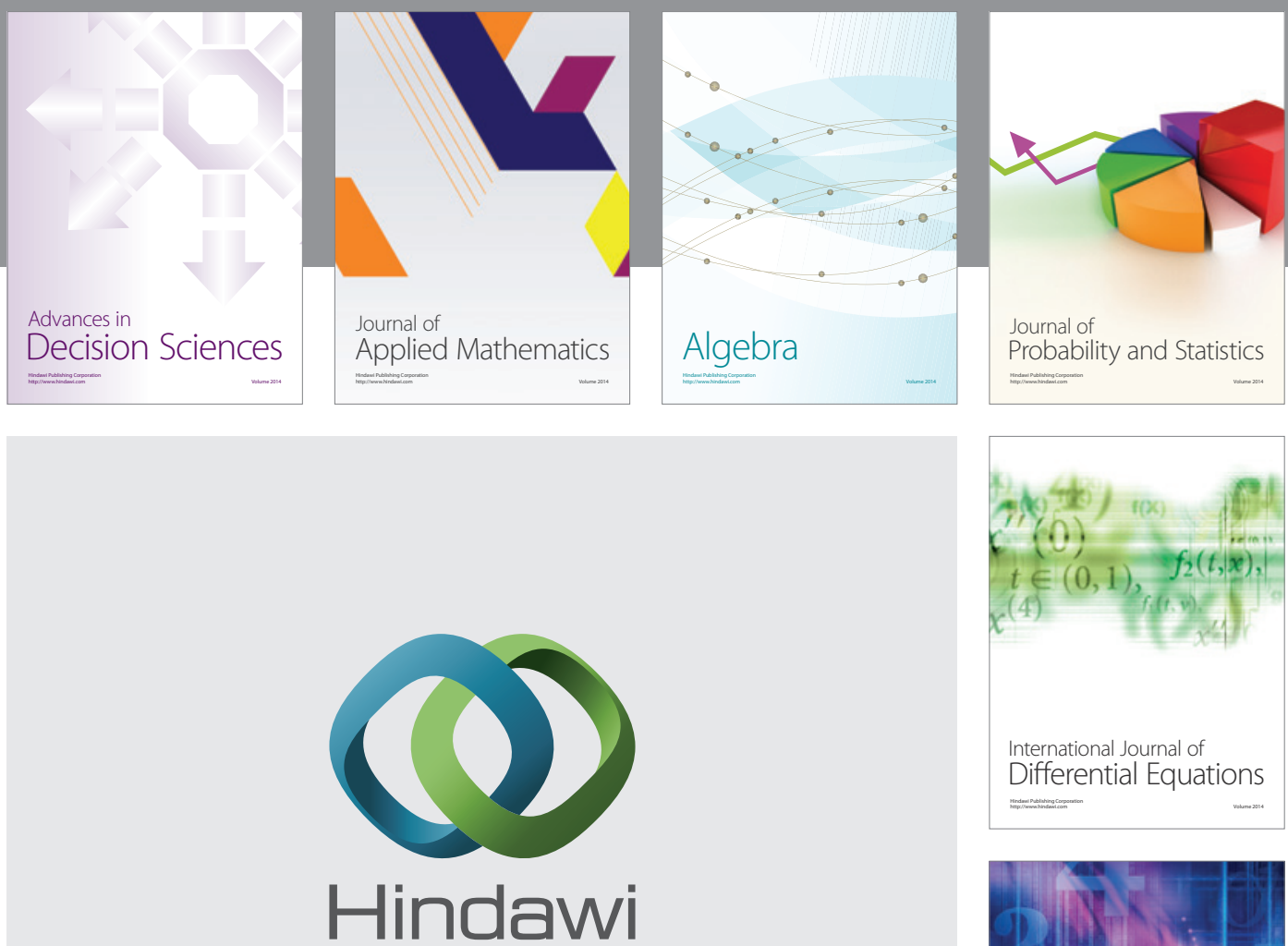

Submit your manuscripts at http://www.hindawi.com
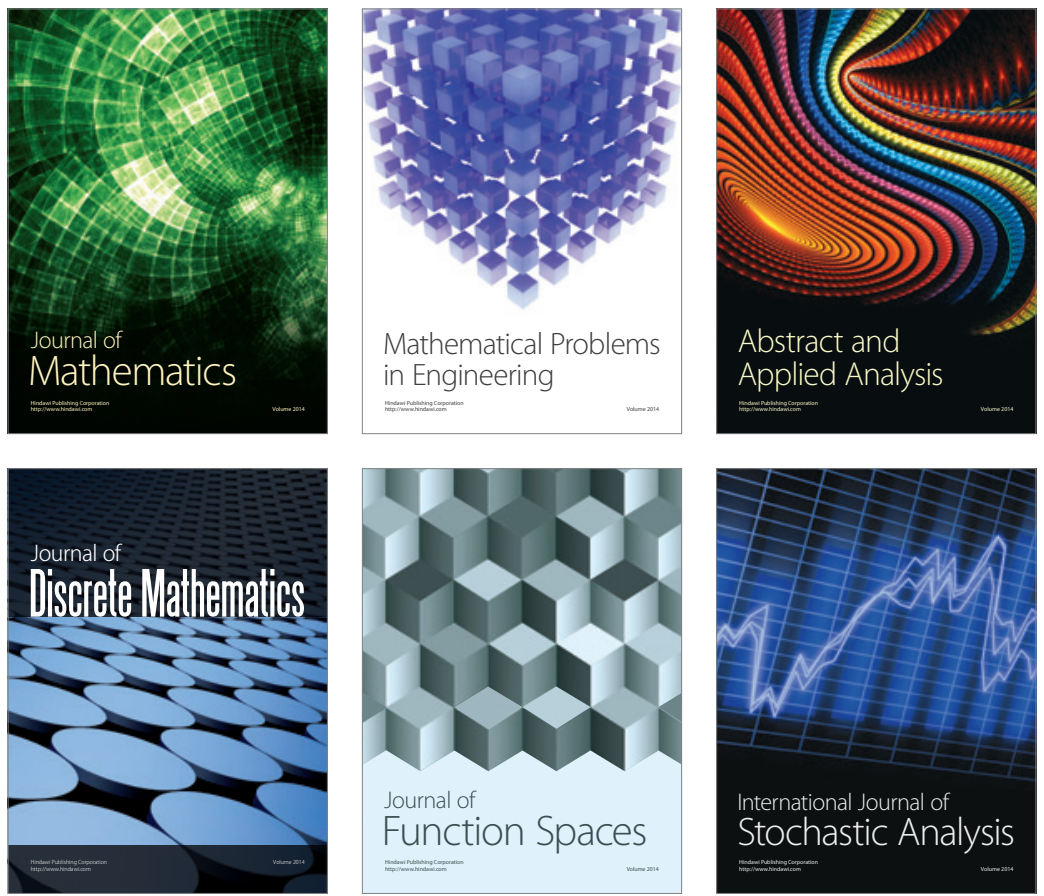

Journal of

Function Spaces

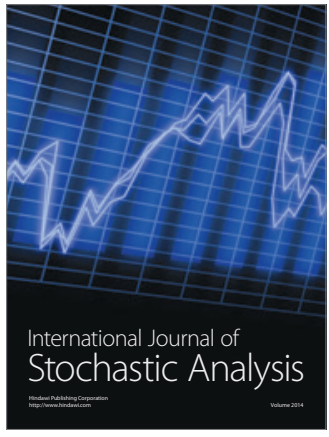

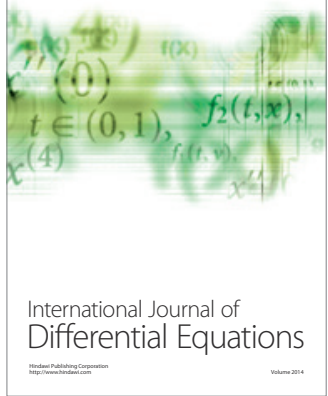
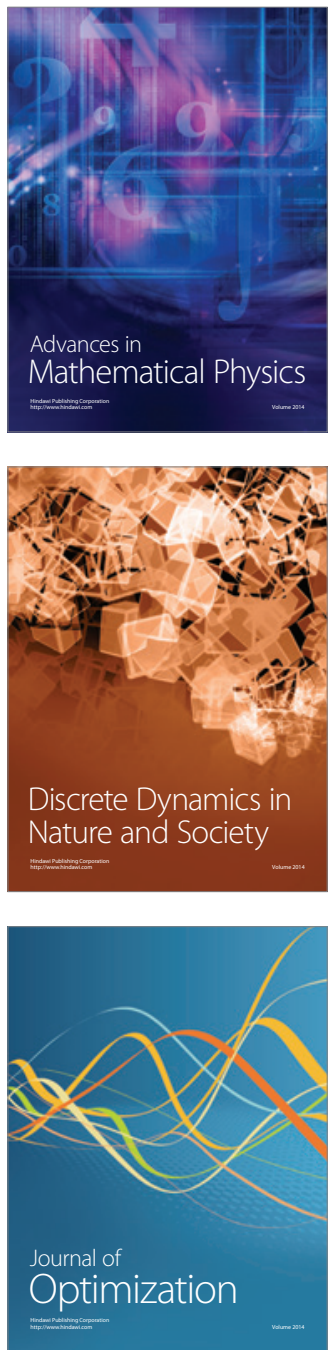